\title{
Characterizing left ventricular mechanical and electrical activation in patients with normal and impaired systolic function using a non-fluoroscopic cardiovascular navigation system
}

Citation for published version (APA):

Piorkowski, C., Arya, A., Markovitz, C. D., Razavi, H., Jiang, C., Rosenberg, S., Breithardt, O-A., Rolf, S., John, S., Kosiuk, J., Huo, Y., Doring, M., Richter, S., Ryu, K., Gaspar, T., Prinzen, F. W., Hindricks, G., \& Sommer, P. (2018). Characterizing left ventricular mechanical and electrical activation in patients with normal and impaired systolic function using a non-fluoroscopic cardiovascular navigation system. Journal of Interventional Cardiac Electrophysiology, 51(3), 205-214. https://doi.org/10.1007/s10840-018-0317-3

Document status and date:

Published: 01/04/2018

DOI:

10.1007/s10840-018-0317-3

Document Version:

Publisher's PDF, also known as Version of record

Document license:

Taverne

Please check the document version of this publication:

- A submitted manuscript is the version of the article upon submission and before peer-review. There can be important differences between the submitted version and the official published version of record. People interested in the research are advised to contact the author for the final version of the publication, or visit the DOI to the publisher's website.

- The final author version and the galley proof are versions of the publication after peer review.

- The final published version features the final layout of the paper including the volume, issue and page numbers.

Link to publication

\footnotetext{
General rights rights.

- You may freely distribute the URL identifying the publication in the public portal. please follow below link for the End User Agreement:

www.umlib.nl/taverne-license

Take down policy

If you believe that this document breaches copyright please contact us at:

repository@maastrichtuniversity.nl

providing details and we will investigate your claim.
}

Copyright and moral rights for the publications made accessible in the public portal are retained by the authors and/or other copyright owners and it is a condition of accessing publications that users recognise and abide by the legal requirements associated with these

- Users may download and print one copy of any publication from the public portal for the purpose of private study or research.

- You may not further distribute the material or use it for any profit-making activity or commercial gain

If the publication is distributed under the terms of Article 25fa of the Dutch Copyright Act, indicated by the "Taverne" license above, 


\title{
Characterizing left ventricular mechanical and electrical activation in patients with normal and impaired systolic function using a non-fluoroscopic cardiovascular navigation system
}

\author{
Christopher Piorkowski ${ }^{1} \cdot$ Arash Arya $^{2} \cdot$ Craig D. Markovitz $^{3} \cdot$ Hedi Razavi $^{3}$ - Chunlan Jiang ${ }^{3}$ - Stuart Rosenberg ${ }^{3}$. \\ Ole-A. Breithardt ${ }^{4}$. Sascha Rolf ${ }^{5}$. Silke John ${ }^{2}$. Jedrzej Kosiuk ${ }^{2} \cdot$ Yan Huo $^{1} \cdot$ Michael Döring $^{2} \cdot$ Sergio Richter $^{2}$. \\ Kyungmoo Ryu ${ }^{3} \cdot$ Thomas Gaspar $^{1} \cdot$ Frits W. Prinzen ${ }^{6} \cdot$ Gerhard Hindricks $^{2} \cdot$ Philipp Sommer $^{2}$
}

Received: 19 December 2017 / Accepted: 22 January 2018 / Published online: 31 January 2018

(C) Springer Science+Business Media, LLC, part of Springer Nature 2018

\begin{abstract}
Purpose Cardiac disease frequently has a degenerative effect on cardiac pump function and regional myocardial contraction. Therefore, an accurate assessment of regional wall motion is a measure of the extent and severity of the disease. We sought to further validate an intra-operative, sensor-based technology for measuring wall motion and strain by characterizing left ventricular (LV) mechanical and electrical activation patterns in patients with normal (NSF) and impaired systolic function (ISF).

Methods NSF $(n=10$; ejection fraction $=62.9 \pm 6.1 \%)$ and ISF $(n=18$; ejection fraction $=35.1 \pm 13.6 \%)$ patients underwent simultaneous electrical and motion mapping of the LV endocardium using electroanatomical mapping and navigational systems (EnSite ${ }^{\mathrm{TM}} \mathrm{NavX}^{\mathrm{TM}}$ and MediGuide ${ }^{\mathrm{TM}}$, Abbott). Motion trajectories, strain profiles, and activation times were calculated over the six standard LV walls.

Results NSF patients had significantly greater motion and systolic strains across all LV walls than ISF patients. LV walls with low-voltage areas showed less motion and systolic strain than walls with normal voltage. LV electrical dyssynchrony was significantly smaller in NSF and ISF patients with narrow-QRS complexes than ISF patients with wide-QRS complexes, but mechanical dyssynchrony was larger in all ISF patients than NSF patients. The latest mechanical activation was most often the lateral/posterior walls in NSF and wide-QRS ISF patients but varied in narrow-QRS ISF patients.

Conclusions This intra-operative technique can be used to characterize LV wall motion and strain in patients with impaired systolic function. This technique may be utilized clinically to provide individually tailored LV lead positioning at the region of latest mechanical activation for patients undergoing cardiac resynchronization therapy.

Clinical trial registration URL: http://www.clinicaltrials.gov. Unique identifier: NCT01629160.
\end{abstract}

Keywords Left ventricular wall motion · Left ventricular strain · Electrophysiology mapping · Dyssynchrony · Cardiac resynchronization therapy

Christopher Piorkowski

Christopher.Piorkowski@herzzentrum-dresden.com

1 Department of Invasive Electrophysiology, University of Dresden-Heart Center, Dresden, Germany

2 Department of Electrophysiology, University of Leipzig-Heart Center, Leipzig, Germany

3 Abbott, Sylmar, CA, USA

4 Agaplesion Diakonie Kliniken, Kassel, Germany

5 DRK Kliniken Berlin Westend, Berlin, Germany

6 Department of Physiology, Cardiovascular Research Institute Maastricht, Maastricht University, Maastricht, The Netherlands

\section{Introduction}

Cardiac disease often has a degenerative effect on cardiac pump function and regional myocardial contraction. An accurate assessment of regional wall motion is therefore a measure of the extent and severity of the disease. Clinically, measurement of cardiac wall motion, strain, and activation patterns can potentially help to better target distinct therapies; for example, targeting of the left ventricular (LV) lead at a location of late mechanical activation in patients undergoing cardiac resynchronization therapy (CRT). Existing imaging-guided methods of wall motion assessment, including echocardiography (echo) and magnetic resonance imaging (MRI), have 
inherent accuracy limitations and are performed remote from an actual interventional working environment. Therefore, development of an intra-operative method to measure regional cardiac activation is advantageous.

Previously, we introduced a novel technique for intraoperative characterization of LV wall motion through the use of an electroanatomical mapping and navigational system, the MediGuide ${ }^{\mathrm{TM}}$ system (Abbott, St. Paul, MN) [1]. We established the feasibility of this technology by evaluating electrical and mechanical activation patterns in subjects with normal systolic function, quantifying normal LV wall motion across the six standard LV segments, and characterizing mechanical dyssynchrony and the patient-specific wall of latest mechanical activation. The significance of this technique, however, lies in its ability to intraoperatively quantify $3 \mathrm{D}$ cardiac mechanics in patients with cardiomyopathies such as heart failure and ventricular tachycardia (VT). Therefore, this study had two objectives: first, to further validate the use of the sensor-based tracking technology for intraoperative characterization of regional LV wall motion in patients with impaired systolic function. Secondly, since this technique is able to simultaneously measure focal electrical and mechanical activation, we sought to compare electrical and mechanical activation patterns in patients with normal (NSF) and impaired systolic function (ISF) and in ISF patients with narrow- and wideQRS complexes.

\section{Methods}

\subsection{Patient populations}

Patients indicated for a left-heart ablation procedure for atrial fibrillation (AF), frequent premature ventricular contractions (PVCs), or ventricular tachycardia (VT) were prospectively enrolled at the University of Leipzig-Heart Center, Leipzig, Germany between July 2012 and December 2013. All patients were at least 18 years of age and in sinus rhythm at time of enrollment. Patients with permanent AF and history of heart disease were excluded. The study protocol was approved by the University of Leipzig-Heart Center ethics committee and the Federal Institute for Drugs and Medical Devices in Germany (BfArM). All patients provided informed consent. Patients were divided into two groups for analysis: those with NSF and those with ISF, classified as such due to the presence of ischemic or non-ischemic cardiomyopathy and an ejection fraction below 55\%. ISF patients were further divided based on QRS width, with a cutoff set at $130 \mathrm{~ms}$, for certain analyses.

\subsection{MediGuide $^{\mathrm{Tm}}$ technology}

The MediGuide ${ }^{\mathrm{TM}}$ system has been described previously [2-5]. Briefly, transmitters integrated in the fluoroscopy c- arm generate a set of low-intensity alternating electromagnetic fields around the patient's chest which allow for real-time visualization of tools embedded with sensors onto live fluoroscopic or pre-recorded cine loops. A reference sensor attached to the patient's sternum automatically compensates the sensor projections for patient movement and variations in respiration. Surface ECG gating is used to compensate for changes in the heart rate. The MediGuide system has proven to be highly reliable and accurate, with a $<0.5-\mathrm{mm}$ offset between the virtual MediGuide Enabled ${ }^{\mathrm{TM}}$ catheter visualization and catheter representation on fluoroscopic cine loops as shown in an independent phantom model study [6]. For this study, in order to characterize $\mathrm{LV}$ wall motion, the $3 \mathrm{D}$ position of a MediGuide sensor was tracked while a MediGuide Enabled $^{\mathrm{TM}}$ catheter was in contact with the LV endocardial wall.

\subsection{Mapping and ablation procedure}

Patient setup before LV motion mapping and the ablation procedure after LV motion mapping were performed per clinical routine. The procedure was performed while the patient was under conscious sedation. Two MediGuide Enabled ${ }^{\mathrm{TM}}$ diagnostic catheters (Livewire ${ }^{\mathrm{TM}}$, MediGuide Enabled ${ }^{\mathrm{TM}}$, Abbott) were placed in the coronary sinus and right ventricular apex to serve as reference signals. Following a single transseptal puncture under fluoroscopy, two contrast-filled ventriculograms (RAO $20-30^{\circ}$ and LAO $50-60^{\circ}$ ) were recorded as a dynamic background for the MediGuide system. After accessing the $\mathrm{LV}$, heparin was administered to maintain an activation clotting time of over $300 \mathrm{~s}$. LV mapping was conducted using either a MediGuide Enabled ${ }^{\mathrm{TM}}$ diagnostic catheter (Livewire ${ }^{\mathrm{TM}}$, MediGuide Enabled ${ }^{\mathrm{TM}}$, Abbott) or ablation catheter (Therapy ${ }^{\mathrm{TM}}$ CoolPath ${ }^{\mathrm{TM}}$ Duo or Safire ${ }^{\mathrm{TM}}$ Duo, MediGuide Enabled ${ }^{\mathrm{TM}}$, Abbott) in conjunction with a steerable transseptal sheath (Agilis ${ }^{\mathrm{TM}}$, Abbott). An electroanatomical mapping system (EnSite ${ }^{\mathrm{TM}} \mathrm{NavX}^{\mathrm{TM}}$, Abbott) connected to the MediGuide system was used to simultaneously collect 12-lead surface ECG and bipolar intracardiac electrograms (IEGMs).

LV motion mapping was conducted non-fluoroscopically during sinus rhythm prior to catheter ablation by placing the roving MediGuide Enabled ${ }^{\mathrm{TM}}$ catheter in continuous contact with the endocardial surface for 15-20 s at multiple locations throughout the LV. Catheter contact was ensured via consistent peak-to-peak amplitude on the bipolar IEGM signal from the roving catheter, while extensive back pressure on the catheter was avoided by checking for elevation of the ST segment on the unipolar IEGM signal from the roving catheter. A homogenous distribution of mapped points throughout the LV was intended for each case, though the specific number of map points collected differed among patients due to variance in LV size and procedure types. Voltage mapping was 
simultaneously performed at each motion mapping location, and low-voltage scar was defined as $<1.5 \mathrm{mV}$.

\subsection{Data pre-processing}

All data analysis was performed offline in Matlab. Raw MediGuide positional data were exported through the EnSite $^{\mathrm{TM}} \mathrm{NavX^{ \textrm {TM } }}$ system and up-sampled from a native sampling frequency of 30 to $101 \mathrm{~Hz}$ to synchronize with electrical signals. Effects of respiration on the raw motion data were compensated for by applying a band-stop filter that excluded frequency components in the range of the observed respiratory rates. For each map point, sinus beats were manually selected for analysis based on consistency of the following electrical and motion characteristics: (1) waveform morphology on at least two surface ECG leads, (2) peak-to-peak voltage amplitude on roving catheter bipolar IEGM signals, (3) cycle length, (4) atrial and ventricular activation depicted on the CS catheter with consistent atrioventricular delays, and (5) MediGuide sensor positional data along all three dimensions. In the case of extra-systolic beats, the beat immediately preceding and immediately following the premature contractions were excluded from analysis. Map points with less than three acceptable beats based on these criteria were excluded from further analysis. Motion and electrical data from the selected beats were ensemble averaged to obtain a representative motion and electrical waveforms per map point, with the onset of the body surface QRS complex serving as the temporal reference.

\subsection{Motion analysis}

Two types of LV wall motion characterizations were performed: point-based displacement and wall segment-based strain. The LV endocardial surface was divided into six standard circumferential wall segments, defining a construct in which each wall segment had two neighboring wall segments. The anatomical boundaries of these wall segments were defined using patient-specific anatomical landmarks, including the centroid of the mitral annulus, the LV apex, and the LV outflow tract. Each map point was then assigned to at least one wall segment, with map points at segmental boundaries between two neighboring wall segments being assigned to both.

\subsubsection{Displacement}

To assess displacement of the LV wall at each map point, ensemble-averaged motion data were used to delineate a 3D trajectory loop. The extent of motion was defined as the integral of distance along the entire 3D path. The extent of motion for all map points within a given wall was averaged together for comparison across walls as well as between patient groups. Similar comparisons were made between the basal, mid- ventricular, and apical regions of the LV. The length of the $\mathrm{LV}$, used as a surrogate for LV size, was determined intraoperatively using the MediGuide system as the distance between the centroid of the mitral annulus to the LV apex.

\subsubsection{Strain calculation and determination of mechanical and electrical activation times}

The process for calculating LV wall strain, which is based on the time-varying change in area of triangles created by connecting individual map points, is described in greater detail in a previous manuscript [1]. The time of mechanical activation for each LV wall segment was defined as the time from the QRS onset to $90 \%$ of the peak minimum of the strain waveform. LV mechanical dyssynchrony was defined as the standard deviation of the times of mechanical activation across the six anatomical LV walls. Similarly, the time of electrical activation at each map point was defined as the time to the first peak of the roving catheter's rectified and ensemble-averaged bipolar IEGM signal above twice the signal's standard deviation. LV electrical dyssynchrony was defined as the standard deviation of the times of electrical activation across the six LV walls.

Mechanical activation patterns were also elucidated using speckle tracking echo for comparison with measurements taken from the MediGuide system. Prior to the ablation procedure, all patients underwent a resting transthoracic 2D speckle tracking echo (GE Vivid 7TM, GE Healthcare, Milwaukee, WI) while the patient was in sinus rhythm. Longitudinal strain waveforms from the basal, mid-ventricular, and apical regions of each of the six standard anatomical LV wall segments (anteroseptal, anterior, lateral, posterior, inferior, and septum) were averaged using Matlab (Mathworks, Natick, MA) to obtain an overall strain waveform for each wall segment. The time of mechanical activation was calculated as the time from the body surface QRS complex to $90 \%$ of the minimum average strain.

\subsection{Statistics}

Continuous variables are reported as mean \pm standard deviation. $p$ values less than 0.05 were considered significant, and a Bonferroni-adjusted $t$ statistic was used for comparisons involving multiple groups.

\section{Results}

\subsection{Patient characteristics}

This study consisted of 28 patients, 10 categorized as NSF patients and 18 categorized as ISF patients, with an age of $62.3 \pm 11.6$ years (NSF, $61.0 \pm 7.4$; ISF, $63.9 \pm 13.7$ years) 
and 23/28 males (NSF, 5/10; ISF, 18/18 males). Patient characteristics and procedural data for the two patient groups are shown in Table 1. NSF patients had significantly larger ejection fractions and narrower QRS complexes than ISF patients and were indicated for radiofrequency ablation of $\operatorname{AF}(n=9)$ or frequent PVCs $(n=1)$. ISF patients had significantly larger left atrial diameters than NSF patients; all had ischemic $(n=$ $16)$ or non-ischemic $(n=2)$ cardiomyopathy and were indicated for radiofrequency ablation of VT $(n=18)$. Of the 18 ISF patients, 7 had narrow-QRS and 11 had wide-QRS complexes.

A total of 2491 map points were collected across all 28 patients, for an average of 89.0 points per patient. Of these, $367(14.7 \%)$ map points had less than three acceptable sinus beats and were excluded from further analysis. A total of 653 map points were recorded from NSF patients, with 145 (22.2\%) map points being excluded from analysis for having less than three acceptable sinus beats. A total of 1838 map points were recorded from ISF patients, with 222 (12.1\%) being removed from analysis for having less than three acceptable sinus beats. ISF patients had significantly more mapping points collected during the procedure $(102.1 \pm 16.1$ vs. $65.3 \pm$ 11.0) than NSF patients, primarily due to their larger heart sizes.

\subsection{Wall motion and strain}

The extent of LV wall motion averaged across all points for each patient is plotted relative to the size of their LV in Fig. 1. In NSF patients, the extent of motion showed a linear increase with larger LV size $(r=0.78 ; p<0.01)$, while ISF patients showed no relationship between extent of motion and LV size $(r=0.10 ; p=0.70)$.

Aggregate strain waveforms were successfully calculated for $167 / 168 \mathrm{LV}$ wall segments across the 28 patients, with the inferior wall of one NSF patient having no eligible triangles

Table 1 Patient characteristics

\begin{tabular}{lll}
\hline & $\begin{array}{l}\text { NSF patients } \\
(n=10)\end{array}$ & $\begin{array}{l}\text { ISF patients } \\
(n=18)\end{array}$ \\
\hline Age (years) & $61.0 \pm 7.4$ & $63.9 \pm 13.7$ \\
Sex (No. of males) & $5 / 10$ & $18 / 18$ \\
Body surface area $\left(\mathrm{m}^{2}\right)$ & $1.57 \pm 0.26$ & $1.58 \pm 0.16$ \\
Left atrial diameter (mm) & $39.9 \pm 5.4$ & $47.4 \pm 10.1$ \\
Ejection fraction $(\%)$ & $62.9 \pm 6.1$ & $35.1 \pm 13.6$ \\
QRS width (ms) & $87.4 \pm 7.2$ & $129.1 \pm 38.8$ \\
Type of ablation procedure & AF $(9)$ & VT (18) \\
Type of cardiomyopathy & Incessant PVC (1) & \\
& None & Ischemic (16) \\
Number of map points taken & $65.3 \pm 11.0$ & Non-ischemic (2) \\
\hline
\end{tabular}

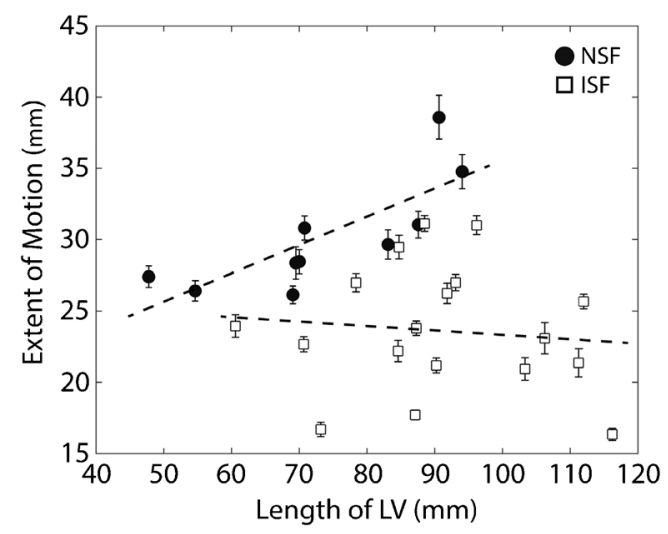

Fig. 1 Extent of motion. Motion pathlengths were averaged across all mapping points in each patient relative to LV length for normal systolic function (NSF; closed circles) and impaired systolic function (ISF; open squares) patients. Linear trend lines are shown through the data of each patient group. Error bars represent the standard error

after applying the criteria. Qualitatively, the majority of strain waveforms exhibited an initial plateau or a limited initial relaxation (positive deflection) followed by a sharp decrease, indicative of systolic contraction, followed by a more gradual increase, indicative of diastolic relaxation. Variations in waveform shape, likely related to averaging strain waveforms across the entire LV wall and/or sparse sampling in certain walls, occurred in less than $15 \%$ of LV wall segments. These variations include waveforms with multiple minimum peaks and those with prolonged minimum peaks. Examples of aggregate strain waveforms and voltage maps from representative NSF and ISF patients are shown in Fig. 2.

The extent of motion averaged across LV walls and regions for NSF and ISF patients are shown in Fig. 3a, b. In NSF patients, the lateral wall showed the greatest extent of motion $(31.3 \pm 7.0 \mathrm{~mm})$ and the septal wall showed the least extent of motion $(28.7 \pm 9.7 \mathrm{~mm})$, though none of the walls had a significantly different extent of motion from the others. Additionally, the extent of motion decreased consistently and significantly from the base to the mid-ventricle to the apex (31.2 $\pm 7.6,29.2 \pm 7.7$, and $26.1 \pm 6.9 \mathrm{~mm}$, respectively). In ISF patients, the anterior, lateral, and posterior walls showed the greatest extent of motion $(24.4 \pm 8.3,24.4 \pm 7.7$, and 24.2 $\pm 7.2 \mathrm{~mm}$, respectively) and the septal wall showed the least extent of motion $(22.2 \pm 7.4 \mathrm{~mm})$, significantly less than the anterior, lateral, and posterior walls. There was little difference in extent of motion across the base, mid-ventricle, and apex in ISF patients $(23.6 \pm 7.4,24.0 \pm 8.1$, and $23.1 \pm 8.4 \mathrm{~mm}$, respectively). NSF patients had a significantly greater extent of motion in all six LV walls and three LV regions than the corresponding wall or region of ISF patients.

Minimum peak values of the aggregate strain waveforms averaged for each wall segment of NSF and ISF patients are shown in Fig. 3c. Larger negative values of systolic strain denote greater changes in the area of triangles connecting motion points, thereby representing more pronounced 
(a)
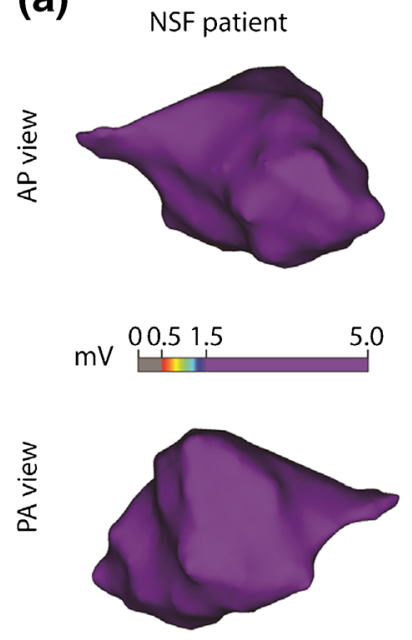

(b)

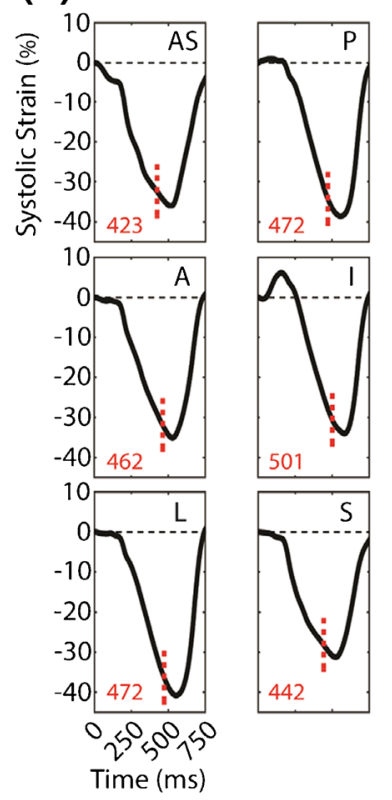

Elect. dyssynchrony $=8.6 \mathrm{~ms}$

Mech. dyssynchrony $=26.9 \mathrm{~ms}$
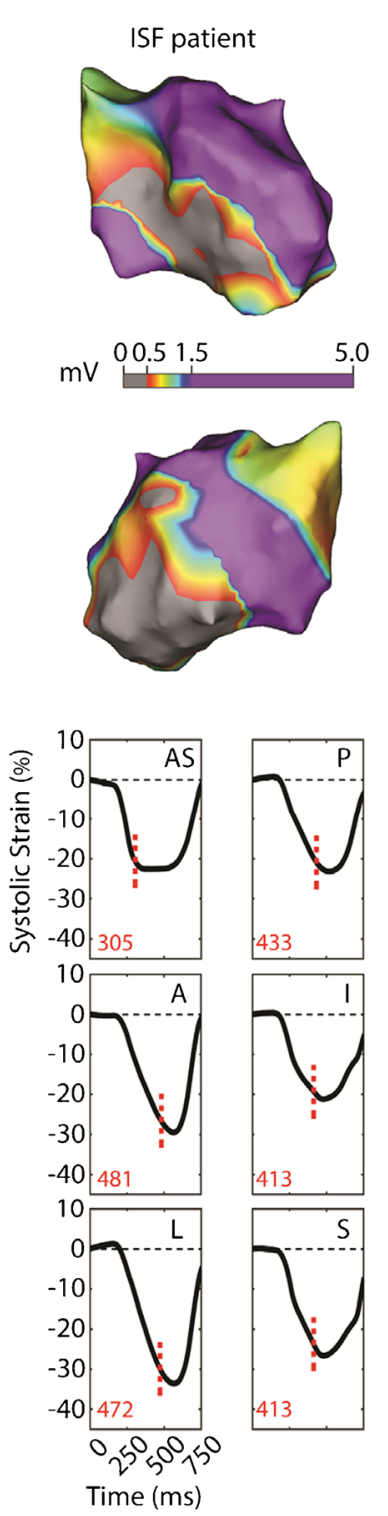

Elect. dyssynchrony $=19.2 \mathrm{~ms}$ Mech. dyssynchrony $=63.1 \mathrm{~ms}$

Fig. 2 Examples of LV voltage maps and strain waveforms from representative patients. Voltage maps (a) are displayed in the anteriorposterior (AP) and posterior-anterior (PA) views, with normal tissue shown in purple, scar tissue $(<0.5 \mathrm{mV})$ shown in gray, and areas of low voltage $(0.5-1.5 \mathrm{mV})$ shown in varying colors. Aggregate strain waveforms (b) are averaged over each LV wall. The red dotted lines represent the activation time, defined as the time at $-90 \%$ of the minimum strain. The electrical and mechanical dyssynchrony, measured as the standard deviation of activation times across the six LV walls, are labeled for each patient (NSF, normal systolic function; ISF, impaired systolic function)

shortening. All walls exhibited substantial shortening with an average of $-40.8 \pm 7.6 \%$ minimum area strain for NSF patients and $-28.5 \pm 8.2 \%$ minimum area strain for ISF patients. In the NSF patients, the posterior $(44.0 \pm 4.4 \%)$ and lateral $(43.6 \pm 5.9 \%)$ walls had the most pronounced shortening

while the anteroseptal $(38.1 \pm 9.6 \%)$ and septal walls $(38.5 \pm$ $7.6 \%$ ) tended to shorten less, though none of the LV walls were significantly different from each other. In the ISF patients, the anterior wall $(31.0 \pm 8.9 \%)$ tended to have the largest shortening while the posterior wall $(24.7 \pm 7.0 \%)$ had the smallest shortening, though again none of the LV walls were significantly different from each other. NSF patients showed significantly more pronounced shortening across all six LV walls than the corresponding wall in the ISF patients.

\subsection{Electrical and mechanical activation patterns}

Within ISF patients, LV walls were separated based on presence of low-voltage areas $(<1.5 \mathrm{mV})$, and the extent of motion and systolic strain were compared between LV walls in NSF patients and LV walls in ISF patients with normal and low voltage as shown in Fig. 4. NSF patients had significantly greater extent of motion than all ISF LV walls, regardless of presence of low-voltage area. Within ISF patients, LV walls with low voltage had significantly less extent of motion than walls with normal voltage. Similarly, NSF patients had significantly more pronounced shortening than ISF LV walls with normal and low voltage. In addition, ISF LV walls with normal voltage trended towards having more pronounced shortening than ISF LV walls with low voltage, though this was not statistically significant.

The average electrical and mechanical dyssynchrony values for NSF, narrow-QRS ISF patients, and wide-QRS ISF patients are shown in Fig. 5. The NSF and ISF patients with narrow QRS had significantly less electrical dyssynchrony than the ISF patients with wide QRS (NSF, 9.0 \pm 5.6 ; narrow-QRS ISF, 10.7 \pm 8.8; wide-QRS ISF, $26.5 \pm 11.9 \mathrm{~ms}$ ) but were not significantly different from each other. The mechanical dyssynchrony, however, showed a different result. When using the MediGuide system, NSF patients showed significantly less mechanical dyssynchrony than both ISF patients with narrow-QRS and ISF patients with wide QRS (NSF, 36.3 $\pm 19.9 \mathrm{~ms}$; narrowQRS ISF, 76.6 $\pm 21.3 \mathrm{~ms}$; wide-QRS ISF, $56.3 \pm 13.6 \mathrm{~ms})$. The mechanical dyssynchrony of the two ISF patient groups were not significantly different from each other. When using echo, the same general trend was shown, with ISF patients with both narrow and wide-QRS complexes having larger mechanical dyssynchrony than NSF patients (NSF, 34.7 $\pm 27.4 \mathrm{~ms}$; narrowQRS ISF, 58.1 $\pm 31.6 \mathrm{~ms}$; wide-QRS ISF, 76.4 $\pm 39.6 \mathrm{~ms}$ ). However, only the wide-QRS ISF patients had statistically significantly more mechanical dyssynchrony than the NSF patients.

The wall of the latest mechanical activation as measured intra-operatively with the MediGuide system and preoperatively with echo are plotted in Fig. 6. The two different techniques produced similar results. In a majority of NSF patients, the lateral and posterior walls were the last to be mechanically activated. Similarly, in wide-QRS ISF patients, the lateral and posterior walls were also most often the walls of 
Fig. 3 Motion and strain in different LV walls and regions. The extent of motion averaged across LV walls (a) and regions (b) and the minimum systolic strain (c) across LV walls are shown for normal systolic function (NSF; closed circles) and impaired systolic function (ISF; open squares) patients.

Distributions significantly different between NSF and ISF patients are indicated with an asterisk, and significant differences between walls or regions within the same patient group are indicated with a cross. Minimum systolic strain is defined as the minimum value of the aggregate strain waveform averaged across LV walls. Larger negative values indicate more pronounced shortening in the given wall. Error bars represent the standard error to improve visualization (a)

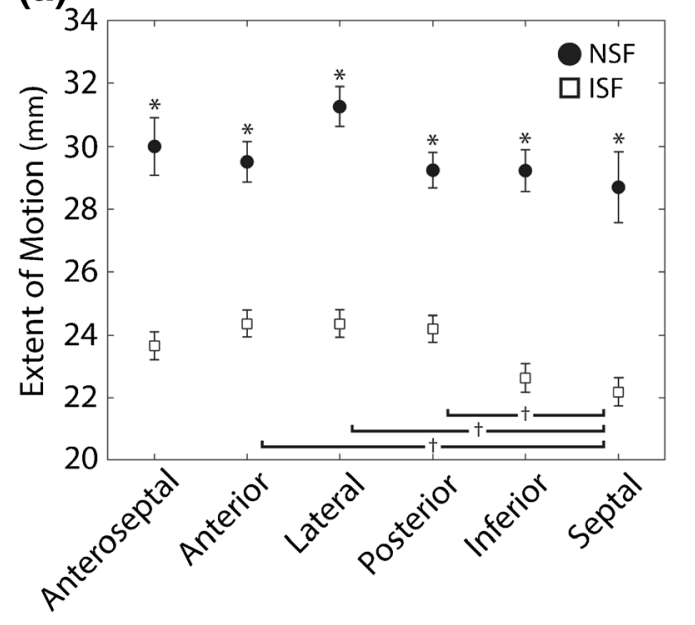

(b)

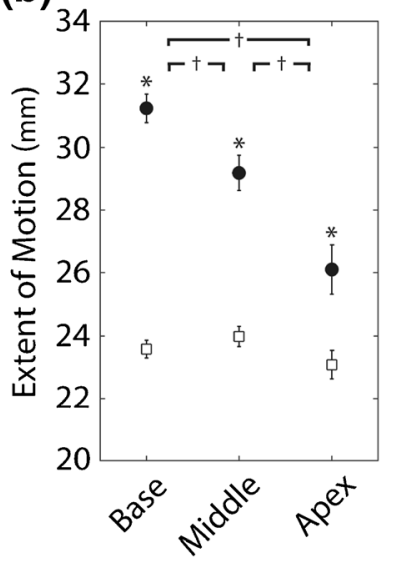

Left Ventricular Region

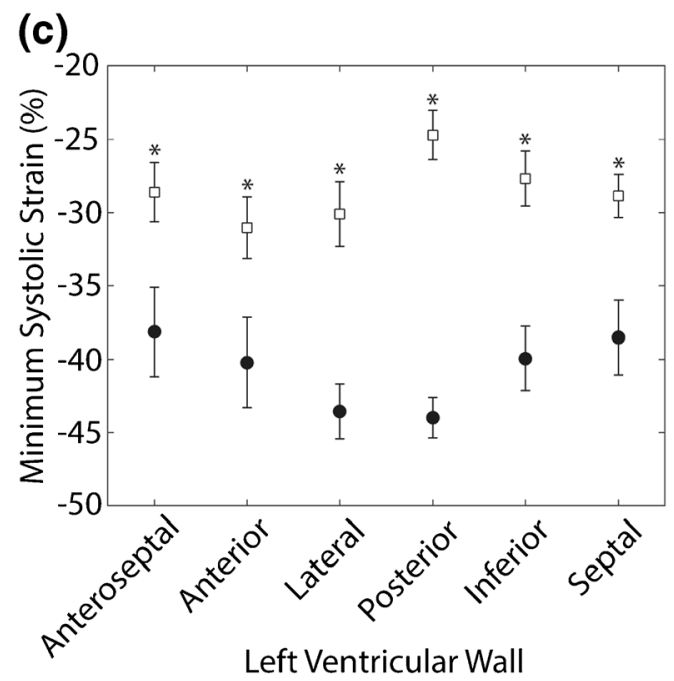

latest activation. However, in narrow-QRS ISF patients, there was no clear pattern as to which wall was the latest to mechanically activate.

\section{Discussion}

In this study, we demonstrated that the MediGuide navigational system can be utilized to characterize and differentiate LV wall motion and strain patterns in patients with multiple etiologies, including those with impaired systolic function undergoing ablation of VT. Given that this technique simultaneously measures both electrical and mechanical activation via the EnSite NavX and MediGuide systems, respectively, it can provide interesting insights into how the two measurements compare across different patient populations.
Fig. 4 Effect of low-voltage areas on LV motion and systolic strain. The extent of motion (left) and minimum systolic strain (right) are shown for patients with normal systolic function (NSF; closed circles) and patients with impaired systolic function (ISF; open squares). Data for ISF patients was separated into LV walls with normal and low voltage (V), defined as $<1.5 \mathrm{mV}$
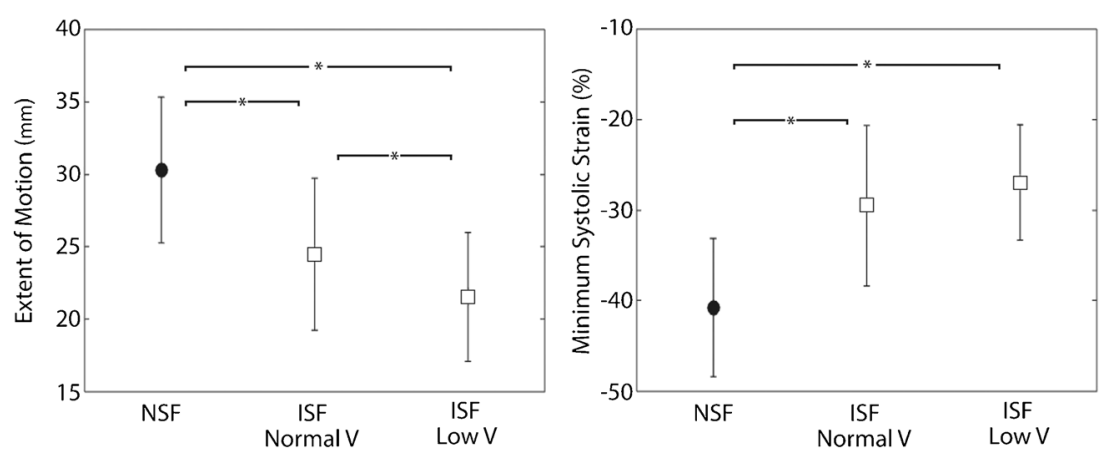


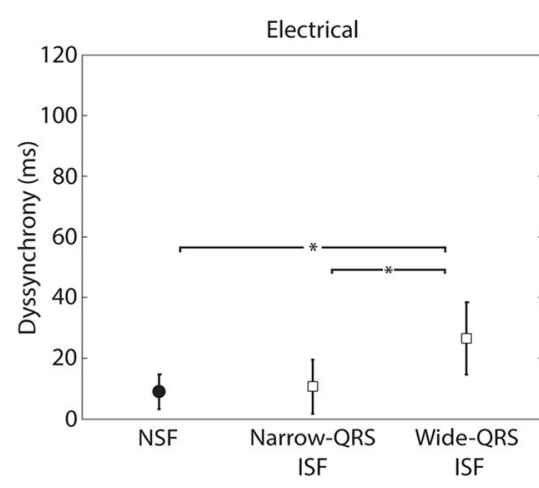

Fig. 5 Electrical and mechanical dyssynchrony. The electrical and mechanical dyssynchrony across LV walls are shown for patients with normal systolic function (NSF; closed circles) and patients with impaired systolic function (ISF; open squares) with narrow- and wide-QRS complexes. Dyssynchrony is defined as the standard deviation of

\subsection{Extent of motion and strain waveforms}

The idea that the MediGuide system can accurately capture LV wall motion is supported by several observations. First of all, motion was larger in NSF patients than in ISF patients, fitting with the lower ejection fraction in the latter. Secondly, NSF patients showed progressively more LV wall motion with larger hearts, which might be understood by the fact that normal hearts will have a close range of ejection fraction, and thus in larger hearts the absolute motion is larger. The fact that this does not hold true in ISF patients may be because in these patients, cardiac enlargement may be linked to worse cardiac function. Thirdly, the NSF patients demonstrated a gradual decrease of motion from the base to the apex, which fits with data from a previous study utilizing $3 \mathrm{D}$ speckle tracing echo [7].

Similar to the motion profiles, strain waveform morphologies were generally more irregular in ISF patients than NSF

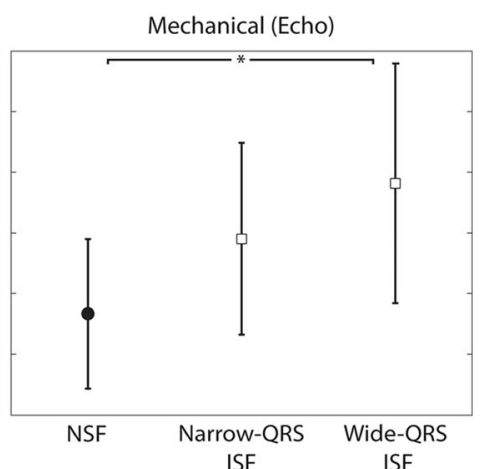

activation times across the six LV walls. Mechanical dyssynchrony was measured by the MediGuide system intra-operatively and using echocardiography pre-operatively. Significant differences in dyssynchrony are indicated with an asterisk. Error bars represent the standard deviation

patients, which may be a direct consequence of the systolic dysfunction. Additionally, the degree of myocardial shortening varied in a non-systematic manner across the LV walls in ISF patients, while NSF patients showed a steady decrease in shortening from the lateral and posterior walls to the septal and anteroseptal walls. NSF patients also had significantly more pronounced shortening across all $6 \mathrm{LV}$ walls than ISF patients. These results not only further validate the accuracy of the proposed technique, but also indicate that the larger extent of motion for NSF patients shown in Fig. 3a, b translates to larger strain values.

\subsection{Electrical and mechanical activation comparison}

NSF patients showed a significantly greater extent of motion and more pronounced shortening than ISF patients, even when the LV walls were separated based on presence of low voltage. This may be attributed to the fact that scar tissue will not only
Fig. $6 \mathrm{LV}$ wall of latest mechanical activation. The LV wall of latest mechanical activation as measured intraoperatively by the MediGuide system (top row) and preoperatively by echocardiography (bottom row) are shown in patients with normal systolic function (NSF) and patients with impaired systolic function (ISF) with narrow- and wide-QRS complexes

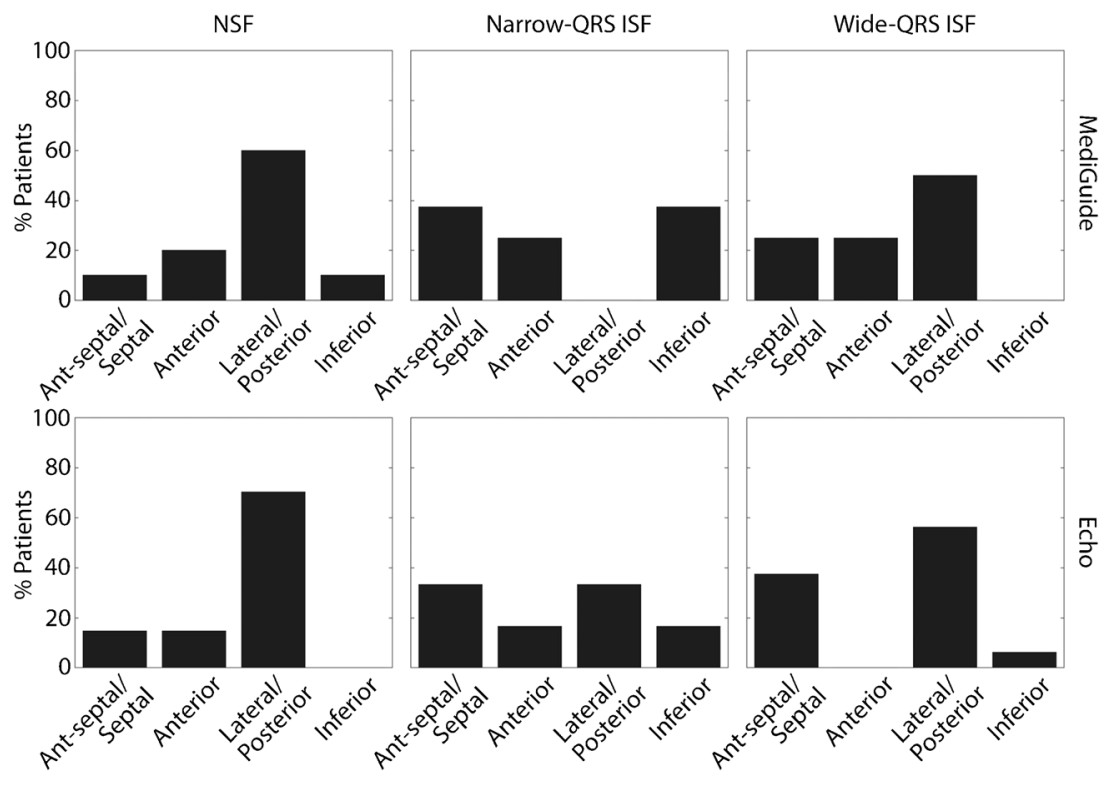


affect the focal region it occupies, but also has a global effect on the function of the entire ventricle. As further evidence, while the extent of motion, which is a more focal measure of activation, was significantly different between ISF LV walls with normal and low voltage, the systolic strain, which is a more global measure of activation, was not significantly different between LV walls with normal and low voltage. A challenge with this type of measurement, however, is that it only incorporates endocardial voltage, excluding scar tissue that may be present on the epicardial surface or within the myocardial wall.

As shown in Fig. 5, NSF patients showed the least amount of both electrical and mechanical dyssynchrony as measured using both the MediGuide system and Echo, as expected. Narrow-QRS ISF patients had significantly more mechanical dyssynchrony than NSF patients when measured using the MediGuide system but not when using Echo. This may be partially attributed to the large variability of the Echo technique in assessing mechanical dyssynchrony, as evidenced by the larger error bars in all three patient groups compared with the measurements using MediGuide. Additionally, wide-QRS ISF patients had significantly more electrical and mechanical dyssynchrony than NSF patients, as would also be expected.

\subsection{Clinical implications for CRT}

The described intra-operative technique to characterize LV wall motion and strain may be useful in guiding LV lead implantation during CRT procedures. As the TARGET and STARTER studies demonstrated, patients receiving targeted LV lead implantation at the point of latest mechanical activation have improved clinical outcomes over patients receiving LV leads using a conventional implantation strategy $[8,9]$. However, the requirement of pre-operative echocardiography makes this difficult to implement in many clinical settings. Using a similar approach as the present study, except taking measurements epicardially instead of endocardially, it may be possible to track a MediGuide sensor-enabled tool such as a guidewire at several locations along the CS tributaries during CRT procedures in order to intra-operatively determine the region of latest mechanical activation and provide guidance for placing the LV lead. This individually-tailored approach to LV lead placement may improve the likelihood of CRT response in the traditional wide-QRS, LBBB patient.

Since several studies have also shown a positive patient response to CRT when the LV lead was implanted at a location with late electrical activation [10-13], the question arises as to whether it matters whether the electrical delay or the mechanical delay is used to guide LV lead implantation. A variable relationship between electrical and mechanical activation has been demonstrated in the literature Wyman et al. found a close relationship between mechanical and electrical timing in dogs during ventricular pacing using direct electrical mapping and
MRI tagging [14]. Similarly, Suever et al. compared cardiovascular magnetic resonance data to electrical measurements directly in CRT patients and found a good correlation [15]. Kroon et al. also found a close relationship in CRT patients, although it was weaker in regions with low voltage [16]. In contrast, Fujiwara et al. evaluated mechanical activation using speckle tracking echo and found a poor relation to electrical measurements in CRT patients [17]. Further, in a study combining computer modeling and speckle tracking echo in CRT candidates, it was shown that the determination of the time of peak shortening can be difficult due to double peaks observed in those signals [18]. Therefore, the more complicated electrical and contraction patterns in failing hearts may explain the variable relationship between electrical and mechanical activation in ISF patients, making the clinical choice between using electrical or mechanical activation non-trivial in heart failure patients undergoing CRT. Since this choice involves practical considerations, such as ease and speed of measurement, the region of latest electrical activation has been more widely adopted clinically. However, with improved intra-operative technology such as this one, implanting the LV lead at the region of latest mechanical activation becomes a more feasible option.

In addition, this technique may re-open the question as to whether heart failure patients with narrow-QRS complexes can benefit from CRT. The largest clinical trial on this topic found that heart failure patients with narrow-QRS complexes $(<130 \mathrm{~ms})$ do not benefit from CRT implantation [19]. Yet, it may be the case that these patients did not benefit from CRT implantation because their LV leads were implanted using the conventional anatomical approach, rather than being optimized for each patient. Results shown in Fig. 5 of the current study and in previous studies indicate that some patients with narrow QRS complexes can still present with mechanical dyssynchrony [20-23]. If we take into consideration the results in Fig. 6, the NSF and wide-QRS ISF patient groups showed a majority of patients with the lateral/posterior walls being the last to be mechanically activated, which is why the conventional LV lead implantation approach targeting the lateral wall makes sense in heart failure patients with wide-QRS complexes. However, the narrow-QRS ISF patients had no clear pattern as to which wall was the last to be activated, which suggests that while they have systolic dysfunction, it is not the same mechanism and presentation as the traditional LBBB wide-QRS patient that has shown clear benefits from CRT implantation. Therefore, it is possible that narrow-QRS patients may benefit from CRT implantation if a patientspecific LV lead implant strategy is executed. Further studies targeting this specific patient group would need to be performed before this can be definitively determined.

\subsection{Limitations}

This was an acute, single-center study with a relatively small number of patients with diverse etiologies. Due to the different 
types of ablation procedures performed, 4 different operators were used for the study. Though motion mapping was performed per the same study protocol, this may have contributed to some of the differences seen across patients. Significantly fewer map points were taken for NSF patients than ISF patients, primarily due to the larger hearts of ISF patients. However, a homogeneous sampling across all walls and regions of the LV endocardium was targeted in all patients. All patients were required to be in sinus rhythm during mapping, which limits future application of this technique.

Funding sources This study was sponsored by Abbott, Inc.

\section{Compliance with ethical standards}

Disclosures $\mathrm{CP}$ has received modest lecture honoraria from Abbott, Biotronik, Boehringer Ingelheim, and Biosense Webster, is a member of the Abbott, Siemens and Biosense Webster advisory boards, and has received research support from Abbott, Biotronik, Imricor, and Philips. CM, CJ, SR, and KR are employed by Abbott with ownership interests. $\mathrm{OAB}$ has received modest lecture honoraria from Abbott, Biotronik, Medtronic and GE Healthcare. S. Rolf and S. Richter have received modest lecture honoraria from Abbott, Biotronik, and Boehringer Ingelheim. FWP serves as a consultant for Abbott and has received research grants from Medtronic, Boston Scientific, EBR Systems, Biological Delivery System Cordis, Abbott, Sorin, Biotronik, MSD, and Proteus Biomedical. GH has received modest lecture honoraria from Abbott, Inc., Biotronik, Medtronic, and Biosense Webster and is a member of the Abbott and Biosense Webster advisory board. PS received modest lecture honoraria from Abbott, Biotronik, Biosense Webster, and Abbott and is a member of the Abbott advisory board. The other authors have no conflicts of interest to disclose.

\section{References}

1. Piorkowski C, Breithardt OA, Razavi H, Nabutovsky Y, Rosenberg SP, Markovitz CD, et al. Mapping-guided characterization of mechanical and electrical activation patterns in patients with normal systolic function using a sensor-based tracking technology. Europace. 2016:euw261. https://doi.org/10.1093/europace/ euw261.

2. Richter S, Doring M, Gaspar T, John S, Rolf S, Sommer P, et al. Cardiac resynchronization therapy device implantation using a new sensor-based navigation system: results from the first human use study. Circulation Arrhythmia and electrophysiology. 2013;6(5): 917-23. https://doi.org/10.1161/CIRCEP.113.000066.

3. Rolf S, John S, Gaspar T, Dinov B, Kircher S, Huo Y, et al. Catheter ablation of atrial fibrillation supported by novel nonfluoroscopic 4D navigation technology. Heart Rhythm: the Official Journal of the Heart Rhythm Society. 2013;10(9):1293-300. https://doi.org/10. 1016/j.hrthm.2013.05.008.

4. Piorkowski C, Hindricks G. Nonfluoroscopic sensor-guided navigation of intracardiac electrophysiology catheters within prerecorded cine loops. Circulation Arrhythmia and Electrophysiology. 2011;4(4):e36-8. https://doi.org/10.1161/CIRCEP.111.962225.

5. Sommer P, Richter S, Hindricks G, Rolf S. Non-fluoroscopic catheter visualization using MediGuide technology: experience from the first 600 procedures. Journal of Interventional Cardiac Electrophysiology: an International Journal of Arrhythmias and
Pacing. 2014;40(3):209-14. https://doi.org/10.1007/s10840-0139859-6.

6. Bourier F, Reents T, Ammar-Busch S, Buiatti A, Grebmer C, Telishevska $\mathrm{M}$, et al. Sensor-based electromagnetic navigation (Mediguide(R)): how accurate is it? A phantom model study. J Cardiovasc Electrophysiol. 2015;26(10):1140-5. https://doi.org/ 10.1111 jee. 12741.

7. Maffessanti F, Nesser HJ, Weinert L, Steringer-Mascherbauer R, Niel J, Gorissen W, et al. Quantitative evaluation of regional left ventricular function using three-dimensional speckle tracking echocardiography in patients with and without heart disease. Am J Cardiol. 2009;104(12):1755-62. https://doi.org/10.1016/j.amjcard. 2009.07.060.

8. Adelstein E, Alam MB, Schwartzman D, Jain S, Marek J, Gorcsan $\mathrm{J}$, et al. Effect of echocardiography-guided left ventricular lead placement for cardiac resynchronization therapy on mortality and risk of defibrillator therapy for ventricular arrhythmias in heart failure patients (from the speckle tracking assisted resynchronization therapy for electrode region [STARTER] trial). Am J Cardiol. 2014;113(9):1518-22. https://doi.org/10.1016/j.amjcard.2014.01. 431.

9. Khan FZ, Virdee MS, Palmer CR, Pugh PJ, O'Halloran D, Elsik M, et al. Targeted left ventricular lead placement to guide cardiac resynchronization therapy: the TARGET study: a randomized, controlled trial. J Am Coll Cardiol. 2012;59(17):1509-18. https://doi. org/10.1016/j.jacc.2011.12.030.

10. Singh JP, Fan D, Heist EK, Alabiad CR, Taub C, Reddy V, et al. Left ventricular lead electrical delay predicts response to cardiac resynchronization therapy. Heart Rhythm: the Official Journal of the Heart Rhythm Society. 2006;3(11):1285-92. https://doi.org/ 10.1016/j.hrthm.2006.07.034.

11. Gold MR, Birgersdotter-Green U, Singh JP, Ellenbogen KA, Yu YH, Meyer TE, et al. The relationship between ventricular electrical delay and left ventricular remodelling with cardiac resynchronization therapy. Eur Heart J. 2011;32(20):2516-24. https://doi.org/10.1093/eurheartj/ehr329.

12. Roubicek T, Wichterle D, Kucera P, Nedbal P, Kupec J, Sedlakova $\mathrm{J}$, et al. Left ventricular lead electrical delay is a predictor of mortality in patients with cardiac resynchronization therapy. Circulation Arrhythmia and Electrophysiology. 2015;8(5):1113-21. https://doi. org/10.1161/CIRCEP.115.003004.

13. Kandala J, Upadhyay GA, Altman RK, Bose A, Heist EK, Mela T, et al. Electrical delay in apically positioned left ventricular leads and clinical outcome after cardiac resynchronization therapy. J Cardiovasc Electrophysiol. 2013;24(2):182-7. https://doi.org/10. 1111/j.1540-8167.2012.02428.x.

14. Wyman BT, Hunter WC, Prinzen FW, McVeigh E. Mapping propagation of mechanical activation in the paced heart with MRI tagging. Am J Physiol-Heart C. 1999;276(3):H881-H91. https://doi. org/10.1152/ajpheart.1999.276.3.H881.

15. Suever JD, Hartlage GR, Magrath RP, Iravanian S, Lloyd MS, Oshinski JN. Relationship between mechanical dyssynchrony and intra-operative electrical delay times in patients undergoing cardiac resynchronization therapy. J Cardiovasc Magn R. 2014;16(1):4. https://doi.org/10.1186/1532-429x-16-4.

16. Kroon W, Lumens J, Potse M, Suerder D, Klersy C, Regoli F, et al. In vivo electromechanical assessment of heart failure patients with prolonged QRS duration. Heart Rhythm: the Official Journal of the Heart Rhythm Society. 2015;12(6):1259-67. https://doi.org/10. 1016/j.hrthm.2015.03.006.

17. Fujiwara R, Yoshida A, Fukuzawa K, Takei A, Kiuchi K, Itoh M, et al. Discrepancy between electrical and mechanical Dyssynchrony in patients with heart failure and an electrical disturbance. Pace. 2014;37(5):576-84. https://doi.org/10.1111/pace.12326.

18. Lumens J, Leenders GE, Cramer MJ, De Boeck BWL, Doevendans PA, Prinzen FW, et al. Mechanistic evaluation of echocardiographic 
dyssynchrony indices patient data combined with multiscale computer simulations. Circ-Cardiovasc Imag. 2012;5(4):491-9. https:// doi.org/10.1161/Circimaging.112.973446.

19. Ruschitzka F, Abraham WT, Singh JP, Bax JJ, Borer JS, Brugada J, et al. Cardiac-resynchronization therapy in heart failure with a narrow QRS complex. N Engl J Med. 2013;369(15):1395-405. https:// doi.org/10.1056/NEJMoa1306687.

20. van Bommel RJ, Tanaka H, Delgado V, Bertini M, Borleffs CJ, Ajmone Marsan N, et al. Association of intraventricular mechanical dyssynchrony with response to cardiac resynchronization therapy in heart failure patients with a narrow QRS complex. Eur Heart J. 2010;31(24):3054-62. https://doi.org/10.1093/eurheartj/ehq334.

21. Bleeker GB, Schalij MJ, Molhoek SG, Verwey HF, Holman ER, Boersma E, et al. Relationship between QRS duration and left ventricular dyssynchrony in patients with end-stage heart failure. J Cardiovasc Electrophysiol. 2004;15(5):544-9. https://doi.org/10. 1046/j.1540-8167.2004.03604.x.

22. Bleeker GB, Schalij MJ, Molhoek SG, Holman ER, Verwey HF, Steendijk $\mathrm{P}$, et al. Frequency of left ventricular dyssynchrony in patients with heart failure and a narrow QRS complex. Am J Cardiol. 2005;95(1):140-2. https://doi.org/10.1016/j.amjcard. 2004.08.082.

23. Yu CM, Chan YS, Zhang Q, Yip GW, Chan CK, Kum LC, et al. Benefits of cardiac resynchronization therapy for heart failure patients with narrow QRS complexes and coexisting systolic asynchrony by echocardiography. J Am Coll Cardiol. 2006;48(11): 2251-7. https://doi.org/10.1016/j.jacc.2006.07.054. 